
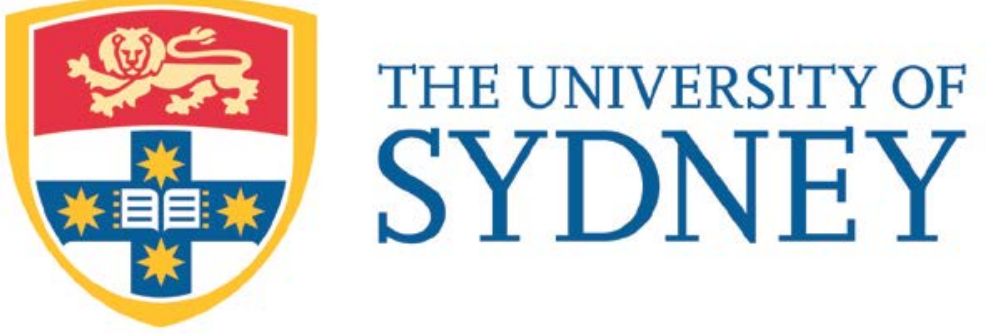

Economics Working Paper Series

$2016-03$

Watercooler chat, organizational structure and corporate culture

Jonathan Newton, Andrew Wait and Simon D. Angus

February 2016 


\title{
Watercooler chat, organizational structure and corporate culture $^{\underline{2}}$
}

\author{
Jonathan Newton $^{\mathrm{a}, *}$, Andrew Wait ${ }^{\mathrm{a}}$, Simon D. Angus ${ }^{\mathrm{b}}$ \\ ${ }^{a}$ School of Economics, University of Sydney. \\ ${ }^{b}$ Department of Economics, Monash University.
}

\begin{abstract}
Modeling firms as networks of employees, occasional collaborative decision making around the office watercooler changes long run employee behavior (corporate culture). The culture that emerges in a given team of employees depends on team size and on how the team is connected to the wider firm. The implications of the model for organizational design are explored and related to empirical research on communication, innovation, the size and decision making of corporate boards and trends in the design of hierarchical structures.
\end{abstract}

Keywords: Shared intentions, hierarchies, teams, delayering, networks, corporate boards.

JEL: C71, C72, C73, D23

\footnotetext{
${ }^{2}$ Follow the latest research at http://sharedintentions.net

*Corresponding author. J.Newton was supported by a Discovery Early Career Researcher Award funded by the ARC (Grant Number: DE130101768).

Email addresses: jonathan.newton@sydney.edu.au (Jonathan Newton), andrew.wait@sydney.edu. au (Andrew Wait), simon. angus@monash.edu (Simon D. Angus) 
Apple is a very disciplined company, and we have great processes. But that's not what it's about. Process makes you more efficient. But innovation comes from people meeting up in the hallways or calling each other at 10.30 at night with a new idea...

- Steve Jobs, founder of Apple Inc. ${ }^{1}$

\section{Introduction}

People talk, share ideas, and collaborate when it is mutually advantageous to do so. Workers bring their collaborative nature with them to the workplace and to their dealings with their colleagues, with whom they interact on shopfloors, in meetings, on production lines and during coffee and lunch breaks. In this paper we consider collaborative decision making in the social environment of the workplace and, using a simple model of adaptive decision making, show that this can have dramatic and far reaching effects on corporate culture and the optimal internal structure of organizations (Figure 1).

Our model takes the well documented fact that humans are particularly good at mutually beneficial collaboration (Tomasello, 2014), and incorporates this fact into a noisy variant (Young, 1998) of the best response dynamic that has been the bread and butter of economic modeling since Cournot (1838). We model firms as networks of employees, each of whom can choose a 'safe' action or a 'risky' action. The risky action represents innovative, even speculative, behavior within the firm. An employee will only find it in his interest to take the risky action if enough of his neighbors in the network do likewise. Within firms, employees are divided into teams. A team is a group of employees who interact together, although they may also interact with others outside of the team. The team represents an employee's work group, department, or even a corporate board or senior management committee (Figure 2).

The ability of employees to engage in collaborative action choice is modeled by the idea of a watercooler, around which small groups of employees within a team can chat and form collaborative intentions. If there are no watercoolers, so that employees cannot share intentions, the model reduces to the canonical model of Young, for which the action profile in which every player chooses the safe action is always a long run equilibrium (Peski, 2010). This result no longer holds when small groups of players can occasionally meet at the watercooler to form shared intentions, coordinating their action choice to their mutual benefit. Instead, by incorporating this basic facet of human nature into the model, we obtain a diversity of behavior, dependent on network topology.

\footnotetext{
1 "The seed of Apple's innovation", BusinessWeek, October 12th, 2004.
} 


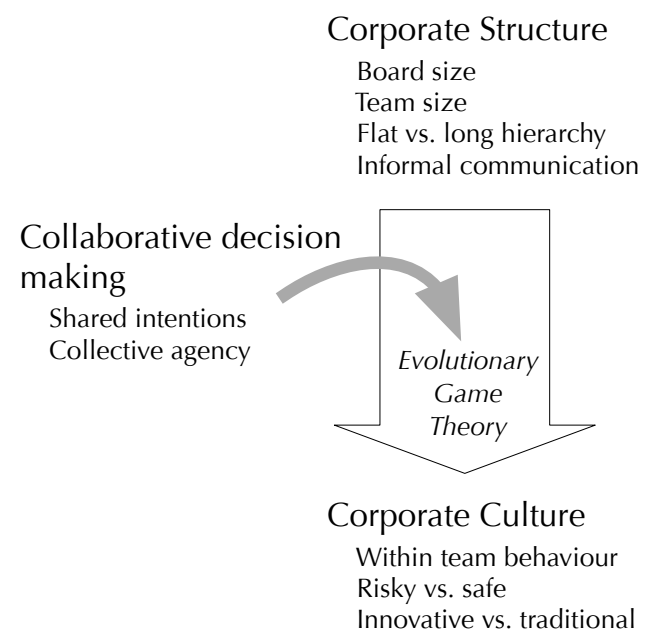

Figure 1: The human ability to share intentions, when combined with the methods of evolutionary game theory, gives predictions about corporate culture and optimal organizational structure.

We find that in order for members of a given team to play the risky/ innovative action in long run equilibrium, some conditions must be satisfied. (i) Firstly, the team must not be too large. The larger a team is, the less likely it is that a fixed amount of collaborative decision making around the watercooler will have an impact on long run behavior. (ii) Secondly, sufficient numbers of employees must be able to coordinate their strategic choice at the watercooler. That is, communication within the team must be strong enough to generate enough collaboration to overcome the systemic bias in favour of the safe action. (iii) Thirdly, the team must not be so small that the influence of its members' external connections can cause them to play the safe action, or, if the team is indeed that small, then all members' connections outside of the team must be to teams that play the risky/innovative action. That is, the external influence from those outside of the team who play it safe must be limited. These conditions provide guidance for organizational design: they can be used to promote or prevent risky behavior in different parts of an organization. Sections 4 and 5 provide examples related to promoting innovation in large teams, delayering and job rotation.

Each of these conditions helps to explain empirical facts documented in the existing literature. A full discussion of this is deferred to Section 3. Here we summarize. Condition (i) helps explain why companies with smaller boards of directors pursue more risky strategies, obtaining higher but more variable returns. It also provides an explanation for why companies seeking to promote innovation create organizational structures based 


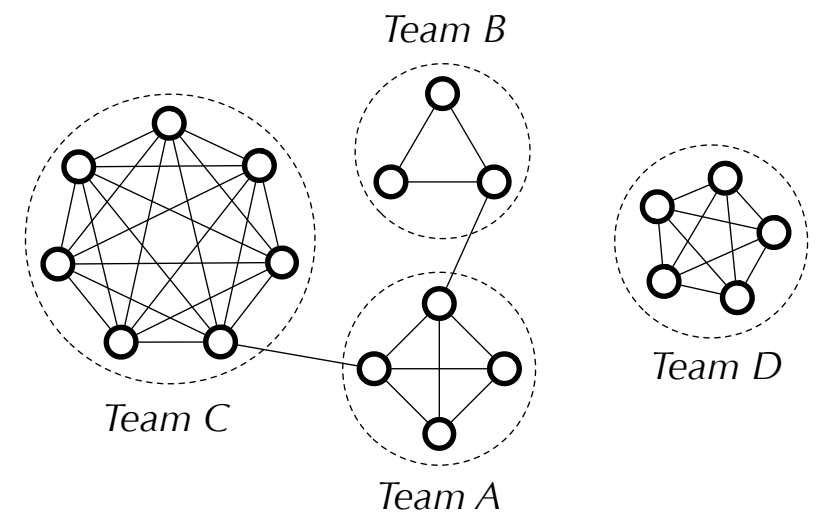

Figure 2: Firms are represented by networks of employees. A line between two employees (vertices) indicates that they interact with some considerable frequency. Every employee interacts with all members of his own team. Some employees in Teams B and C interact with employees in Team A. In contrast, Team D is isolated from the rest of the firm.

around small teams. Condition (ii) helps explain the efforts that firms take to increase spontaneous interaction and facilitate informal communication between workers; that is, to create larger watercoolers. Condition (iii) helps explain why organizations seek to foster independence within teams and even isolate research units from other parts of the organization.

This paper contributes to several strands of literature. The practical contribution is to the literature on the importance of the workplace social environment - the nature and patterns of interaction between workers in a firm (see, for example Bandiera et al., 2005; Gibbons and Henderson, 2013; Kandel and Lazear, 1992). We demonstrate how the facilitation of collective agency by the workplace social environment can have a significant effect. To do this we turn to the literature on adaptive decision making and evolution. By their nature, evolutionary models often focus on long run equilibria. This is similar to how the relational-contracting literature adapts long run folk theorems to study firms (Baker et al., 1999; Levin, 2003; Li et al., 2014), the difference being that evolutionary models impose very low rationality requirements on agents. Such low rationality models have had success at explaining laboratory data (Chong et al., 2006) as well as empirical phenomena as diverse as crop-sharing norms (Young and Burke, 2001) and the wearing of the Islamic veil (Carvalho, 2013). The current paper shows how the incorporation of collective agency into such models can lead to even richer empirical predictions whilst retaining the simplicity and elegance of evolutionary methodology.

The incorporation of collective agency into perturbed evolutionary dy- 
namics is a relatively new and rapidly growing literature (Newton, 2012a,b; Newton and Angus, 2015; Sawa, 2014; Serrano and Volij, 2008), although considerable work has been done in the context of matching, where pairwise deviations represent intentional behavior by coalitions of size two (Jackson and Watts, 2002; Klaus et al., 2010; Klaus and Newton, 2016; Nax and Pradelski, 2014; Newton and Sawa, 2015). The proclivity of humans to engage in collective agency is well documented ${ }^{2}$ and recent research in developmental psychology has shown that the urge to collaborate is a primal one, manifesting itself from ages as young as 14 months (Tomasello, 2014; Tomasello et al., 2005; Tomasello and Rakoczy, 2003). Recent theoretical work has shown that the ability to act as a plural agent will evolve in a wide variety of situations (Angus and Newton, 2015; Bacharach, 2006; Newton, 2015). The authors of the current paper believe that the evidence in favour of the incorporation of collective agency into models of human behavior is overwhelming. Furthermore, adaptive/evolutionary models are ideal for this as, in contrast to static analyses, they provide explicit models of behavior both in and out of equilibrium.

Finally, we note that work on collective agency in evolutionary dynamics builds on a broader literature on coalitional behavior in game-theoretic models. The concept of joint optimization underpins cooperative game theory (See Peleg and Sudholter, 2003, for a survey) and also motivates a small but established literature at the intersection of noncooperative and cooperative game theory (See, for example Ambrus, 2009; Aumann, 1959; Bernheim et al., 1987; Konishi and Ray, 2003). However, despite the noted limitations of methodological individualism in economics (Arrow, 1994), the use of coalitional concepts in economics has not attained the same level of popularity as, for example, the use of the concept of beliefs, except insofar as the concepts of the household and the firm assume a sharing of intentions on the part of the individuals within those structures. The contrast is interesting, as developmental studies of children indicate that they collaborate at earlier ages than they can understand beliefs. ${ }^{3}$ One of the goals of the current paper is to show how a weakening of methodological individualism can lead to simple and striking economic predictions that flow from some of the deepest currents of human nature.

The paper is organized as follows. Section 2 gives the model. Section 3 studies isolated teams of different sizes. Section 4 considers the firm owner's problem. Section 5 studies corporate hierarchies. Section 6 concludes. All proofs not in the main body of the text are given in the appendix.

\footnotetext{
${ }^{2}$ In the words of Tomasello (2014): “...humans are able to coordinate with others, in a way that other primates seemingly are not, to form a "we" that acts as a kind of plural agent to create everything from a collaborative hunting party to a cultural institution."

${ }^{3}$ See Baron-Cohen (1994); Call and Tomasello (1999); Carpenter et al. (1998a,b); Wellman and Bartsch (1994); Wellman et al. (2001).
} 


\section{Model}

Let $N$ be a finite set of employees. Each employee, $i \in N$, has a set of colleagues with whom he interacts, denoted by $\triangle_{i} \subseteq N \backslash\{i\}$, and any interaction is mutual $j \in \Delta_{i} \Leftrightarrow i \in \Delta_{j}$. This means that the interaction structure can be depicted as an undirected network (Figure 2).

Employees have two possible modes of behavior, either taking a safe or a risky action. Let $X^{t} \subseteq N$ denote the set of employees who take the risky action at time $t$. Each employee prefers to take the risky action if and only if at least a proportion $q>1 / 2$ of his neighbors also take the risky action. For $i \in N$, let $q_{i}(X)$ be the proportion of employee $i$ 's neighbors who are in set $X \subseteq N$.

Just like in many real workplaces, groups of employees occasionally meet at a watercooler. Time is continuous and any given set of employees $C \subseteq N$ meets at the watercooler with Poisson arrival rate $\lambda_{C} \in \mathbb{R}_{\geq 0}$. When $\lambda_{C}=0$, the set of employees $C$ never meet at the watercooler. This could be because they work in different areas of the organization, or because there are too many employees in $C$ for them to comfortably fit around a watercooler at the same time. Let $\mathcal{C}$ denote the set of all groups of employees who meet from time to time:

$$
\mathcal{C}:=\left\{C \subseteq N: \lambda_{C}>0\right\}
$$

Note that standard individualistic dynamics, for example Young (1993) and Kandori et al. (1993), correspond to the restriction that $\mathcal{C}=\{C \subseteq N$ : $|C|=1\}$, that is to say there is no watercooler. In contrast, the current model admits the possibility of decision making by non-singleton sets of employees. In the example of Figure 3(a), we see that the set of employees $C=\{a, b, c\}$ meet at the watercooler.

When employees meet at the watercooler, they have the opportunity to coordinate a change in action. Specifically, when set $C$ meets at the watercooler, subsets of $C$ can switch to the risky action if it is in their mutual best interests to do so. Let $\bar{C}(X, C)$ be the maximal $\hat{C} \subseteq C$ such that $q_{i}((X \backslash C) \cup \hat{C}) \geq q$ for all $i \in \hat{C}$. That is, $\bar{C}(X, C)$ is the largest set of employees within $C$ who, fixing the actions of those outside of $C$, prefer to take the risky action if every other employee in $\bar{C}(X, C)$ also takes the risky action. In the example of Figure $3(\mathrm{a})$, from state $X^{t-}$, if we assume that $q=2 / 3$, then $\bar{C}\left(X^{t-}, C\right)=\{a, b\}$.

If $X^{t-}$ is the state immediately preceding an updating opportunity for some group of employees $C$ at time $t$, let $X^{t}=\left(X^{t-} \backslash C\right) \cup \bar{C}\left(X^{t-}, C\right)$. In other words, the employees in $C$ agree to update their actions in such a way that no subset of employees within $C$ could do better than by following this 

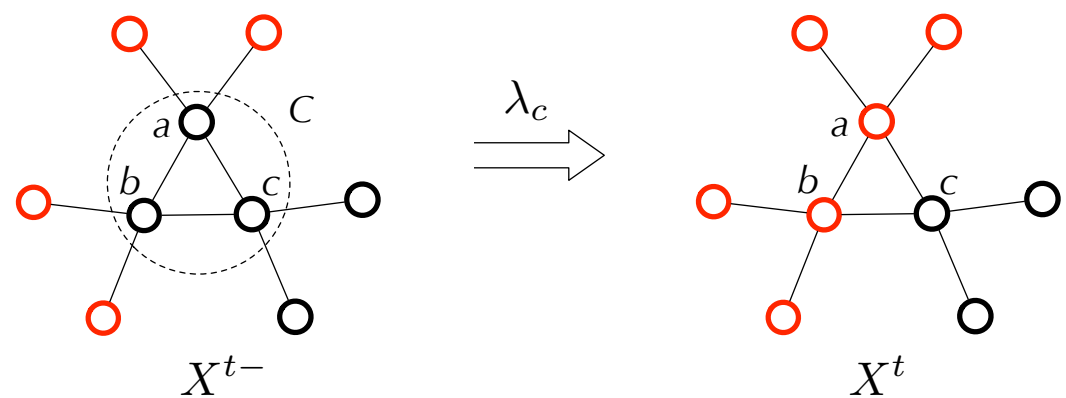

(a) $C=\{a, b, c\}$ meet at the watercooler and $\bar{C}\left(X^{t-}, C\right)=\{a, b\}$ adopt the risky action.
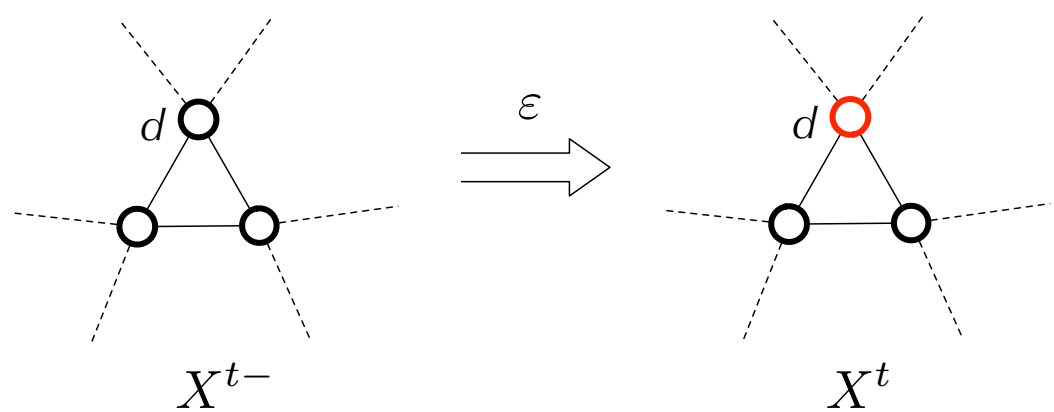

(b) Employee $d$ is hit by a random shock and switches his action.

Figure 3: The strategy updating process. It is assumed that $q=2 / 3$. Employees circled in red are playing the risky action. The remaining employees are playing the safe action.

plan, given that the others within the group also do so. ${ }^{4}$ For the example of Figure 3(a), as $C=\{a, b, c\}$ and $\bar{C}\left(X^{t-}, C\right)=\{a, b\}$, we have that $a, b \in X^{t}$, but $c \notin X^{t}$. Individuals $a$ and $b$ have coordinated a mutually beneficial switch to the risky action. Note that neither $a$ nor $b$ would wish to switch to the risky action if the other were not also switching.

Employee behavior is perturbed by random shocks. With Poisson arrival rate $\varepsilon$, shocks hit the organization. When such a shock occurs, an employee $i \in N$ is selected uniformly at random and his action is flipped to the alternative action. This means that if $i \in X^{t-}$, then $X^{t}=X^{t-} \backslash\{i\}$, and if $i \notin X^{t-}$, then $X^{t}=X^{t-} \cup\{i\}$. This captures employee mistakes, randomness in the translation between intent and action, or simply exogenous influences on behavior. Such a shock is illustrated in Figure 3(b), where employee $d$ is hit by a random shock, causing him to switch from the safe action to the risky action.

\footnotetext{
${ }^{4}$ That is, fix the actions of players outside of $C$ and consider the induced game with player set $C$. The actions chosen by players in $C$ are a strong equilibrium (Aumann, 1959) of the induced game.
} 
Employee behavior is thus determined by a continuous-time Markov process on state space $\mathcal{P}(N)$, with transition probabilities derived from the above description of the process. Note that for $\varepsilon>0$, the process is irreducible and therefore has a unique invariant measure $\pi_{k, q, \varepsilon}$. We are interested in the long run behavior of the process for small values of $\varepsilon$. By standard arguments, as $\varepsilon \rightarrow 0, \pi_{k, q, \varepsilon}$ approaches a limiting measure $\pi_{k, q, 0}$ States with positive weight under $\pi_{k, q, 0}$ are known as stochastically stable states, or long run equilibria of the process. For small values of $\varepsilon$, the process will spend the vast majority of time at such states.

\section{Independent teams and corporate culture}

We start by analyzing the behavior of a single team within a firm in isolation. Within the team, each agent interacts with every other team member. That is, we are interested in a team like Team D in Figure 2, in which no employee interacts with anyone from outside of their own team, but there is a complete network of interaction within the team itself. This assumption - that everyone interacts with every other team member but no one else - is an appropriate simplifying assumption for autonomous work groups, senior management teams or corporate boards, where the number of agents is not so large so that any two individuals never interact, and the bonds within the group are much stronger than connections to outsiders, as formalized by Assumption 1.

Assumption 1. For all $i \in N, \Delta_{i}=N \backslash\{i\}$.

In the model described above, standard individualistic perturbed best response dynamics will always select $X=\emptyset$ as a long run equilibrium, regardless of the size of the team (Kandori et al., 1993; Young, 1993). But this extreme view of a firm does not allow any coordinated decision making by employees. As noted in the Introduction, humans are naturally social and collaborative creatures, and these instincts will be brought into the work environment. Accounting for the possibility of collaborative sharing of intentions moves us away from the individualistic outcome, enabling risky behavior to be selected as the unique long run equilibrium provided certain conditions are satisfied.

The development of shared intentions in an organization is aided by frequent social interactions. Such interactions enable employees to discuss their intentions, jointly formulate plans, and get a feel for the work ethic of their colleagues. However, practical considerations constrain how many employees will gather together at any one time. For example, on any given day only a fraction of all employees will meet in the lunchroom or strike up a conversation around the coffee machine. To capture this, let the maximum number of employees who can simultaneously meet at a watercooler be the 
parameter $k$. Thus the set of groups of employees who meet from time to time is exactly the set of groups of employees of size at most $k$.

Assumption 2. $\mathcal{C}=\{C \subseteq N:|C| \leq k\}$ for some $k \in \mathbb{N}_{+}$.

The standard model of individualistic perturbed best response corresponds to $k=1$. In this case, individuals make their decisions in isolation, as if everyone is "working from home" and no one ever interacts in a common workspace or in an office. However, most work environments have some social interaction, allowing workers to meet and make joint decisions about the actions they will take. This possibility can significantly alter the behavior of employees, and hence the performance of firms.

Given the above assumptions, we are in a position to give sufficient conditions for safe or risky behavior to emerge as a unique long run equilibrium.

Theorem 1. Under Assumptions 1, 2, in any long run equilibrium $X$,

$$
\begin{aligned}
& |N|-1 \geq \frac{k}{2 q-1} \quad \Longrightarrow \quad X=\emptyset, \\
& |N|-1 \leq \frac{k-2}{2 q-1} \quad \Longrightarrow \quad X=N .
\end{aligned}
$$

Three comparative statics regarding behavior of agents within firms arise naturally out of the Theorem above. The first of these relates to team size.

Corollary 1. All else equal, large teams are less likely to engage in risky/innovative behavior than small teams.

Holding $k$ and $q$ constant, an increase in the number of people in a team $(N)$ makes it harder to satisfy the condition for $X=N$ but easier to satisfy the condition for $X=\emptyset$. Consequently, the model predicts that, other things equal, we are less likely to observe risky actions taken by larger teams. This prediction is consistent with three strands of evidence: econometric studies, laboratory experiments, and case studies.

Consider first econometric studies of the relationship between the size of corporate boards and financial performance. Eisenberg et al. (1998) identify a significant negative correlation between board size and profitability for small and medium-sized Finnish firms. Similarly, Yermack (1996) finds that companies with higher valuations have smaller boards of directors. Consistent with the prediction of Corollary 1, smaller board size creates an environment in which members are more likely to adopt a risky/innovative strategy, yielding higher average returns. ${ }^{5}$ Considering

\footnotetext{
${ }^{5}$ Coles et al. (2008) find that a smaller board is advantageous for relatively simple firms that are not overly diversified. They also suggest that larger boards play an important advisory role in diversified firms; they estimate firm value (measured by Tobin's $q$ ) is enhanced in diversified firms with larger boards.
} 
variability in returns, Chaganti et al. (1985) find that smaller board size is linked to higher risk of failure for firms in the retail industry. Cheng (2008) reports evidence that firms with larger boards have lower variability of corporate performance. Again, this is consistent with Corollary 1; small boards are more likely to adopt risky strategies, which are associated with higher but more variable returns.

Supportive evidence from the lab examines the relationship between team size and participants' willingness to take an action with a high degree of strategic complementarity (effectively the risky strategy). Van Huyck et al. (1990) study agents' choices of action in a minimum-effort game, where the payoff depends on the lowest level of effort exerted. They find that agents in larger teams are more likely to choose low effort (the safe action) than agents in smaller teams, who are more likely to choose high effort, which is the risky action because it only pays off if all other participants do likewise. These experimental findings are robust to several extensions. For example, Weber et al. (2001) add a leader to the experiment of Van Huyck et al. (1990). The leader can communicate to all the team members before they choose their action, extolling the benefits of cooperation in an attempt to facilitate efficient coordination (where all agents choose high effort). Again, results suggest that coordination on the high effort (risky) equilibrium is harder to sustain in large teams than in small teams.

Evidence from case studies suggests that companies that place a high value on innovation try to create a work environment based around collaboration and interaction within small teams. Stross (1996) notes that Microsoft, "even as it grew large, was deliberately fashioned to perpetuate the identity of small groups". Similarly, Cook (2012) notes that Google, Cisco and Wholefoods have organizational structures founded on small entrepreneurial groups. In particular, the cited study notes that at Google

"they focus on multiple smaller workgroups that may have a project manager overseen by committees. They are very independent. The basic concept inspired by the founders is to maintain an entrepreneurial culture. Google, Inc. views small teams as individual start-ups. Google consists of many startups within a start-up."

The second comparative static relates to the number of employees who can meet and jointly determine their actions.

Corollary 2. All else equal, risky/innovative behavior is more likely at higher values of $k$.

$k$ limits how many employees can informally meet; consequently, it restricts the numbers of workers who can collectively coordinate their decision 
making at any given time. If we are to observe the risky action in long run equilibrium, a sufficient number of employees must be able contemporaneously engage in such joint decision making. This Corollary implies that greater opportunities for larger groups of employees to communicate, share intentions and collaborate should lead to more innovation and risk taking.

Again, there exists supportive econometric, experimental and case study evidence. Gant et al. (2002a) find higher levels of productivity for steel production lines that adopted innovative management practices when workers have denser social networks. Examining data from R\&D firms, Kraut et al. (1990) find that informal communication, aided by physical proximity, is vital for successful innovative collaboration. Cooper et al. (1992) run an experiment of a stag-hunt two player weak-link game, very similar in structure to our model here. In their experiment, without communication, agents choose the inefficient safe strategy. One-way communication, where one agent could signal their intended action, increases the selection of the risky strategy to $87 \%$ by receivers of a message that the sender would opt for the risky action. When two-way communication is permitted, $91 \%$ of teams choose the risky action.

A secondary implication of Corollary 2 is that if a firm values innovative behavior, then it will attempt to increase $k$. In these cases, an organization will wish to create an environment that is conducive to spontaneous interactions between colleagues. Firms sometimes make great efforts to bring employees to the same physical space so as to facilitate informal communication between as many workers as possible. Tech firms such as Google invest in campuses, cafes and hangouts to provide an environment conducive to informal interaction, and the professional services firm KPMG has recently piloted collaborative work areas in some of its offices (Evans, 2015). Organizations can foster informal discussion, increasing $k$, by sponsoring social activities and corporate retreats, or by establishing consultative working groups to broaden employees' networks with their co-workers.

It is conceivable that the value of $k$ within a firm could vary over time, either due to the actions of managers or because of exogenous changes in the environment. Corollary 2 suggests that employees should be more likely to switch to a risky action following periods when the watercooler is 'large' (high $k$ ). Social or cultural events might provide a catalyst for informal discussions between employees; the Monday following the Super Bowl or the final game in the World Series might create an opportunity for conversations between employees to start, conversations that can lead onto work-related topics.

The third comparative static arising from Theorem 1 relates to $q$, the share of an employee's colleagues with whom he interacts who must take the risky action in order for the employee in question to have a preference for taking the risky action over the safe action. 
Corollary 3. All else equal, risky behavior is less likely with a higher value of $q$.

A higher value of $q$ works against the adoption of risky behavior. This is because the higher is $q$, the fewer random shocks are required to move a team from playing 'risky' to playing 'safe', and the more random shocks are required to move the team back in the opposite direction. For higher values of $q$, in order for employees in a team to opt for the risky action in long run equilibrium, teams will have to be correspondingly smaller (lower $N$ ) or the watercoolers correspondingly larger (higher $k$ ). In fact, when $q=1$, to guarantee risky behavior the entire team must be able to fit around the watercooler at the same time.

Factors that determine $q$ include remuneration structures, specifically the apportionment of reward and blame within the firm when things go right and when things go wrong. By definition, engaging in risky/innovative behavior carries a potential downside, and in a corporate environment there are various ways in which a principal could react when such a downside is realized. If failed innovation carries serious blame, then an employee is unlikely to want to take the risk unless a high proportion of his colleagues do likewise. This corresponds to a high $q$. Conversely, if management accepts failure as a necessary side-effect of innovation, then $q$ will be relatively low. As Garry Ridge, the CEO of lubricant manufacturer WD-40 says: "Why waste getting old if you can't get wise? We have no mistakes here, we have learning moments." 6 Similarly, at Google, mistakes are celebrated: the team behind an unsuccessful product launch in 2009 were lauded, given bonuses and even a prize. Eventually the failed product evolved into Google Plus (Srikant, 2014).

\section{The firm owner's problem}

Given an objective such as maximizing firm value or achieving market dominance, a firm owner will wish to build a firm's organizational architecture and physical work environment that is consistent with its strategic objectives. Organizational culture cannot be enforced through a formal contract, but it can be supported and reinforced through workplace design. In the current context this means that, to the extent to which they are choice variables, a firm owner will choose $N, k$ and $q$ so as to ensure that employees play her desired action in long run equilibrium.

It follows from Theorem 1 that it is relatively easier to get large teams to take the safe action and small teams to take the risky action. As a consequence, other things equal, a firm wishing to pursue a risky or safe

\footnotetext{
6 "Leadership Lessons From WD-40's CEO, Garry Ridge" - Forbes Magazine, June 28th, 2011.
} 
strategy should set up small or large work groups, respectively. However, other aspects of the production process may also enter into the firm owner's objective function. Consider a firm owner whose payoff is $|X|$; that is, she wishes to have as many employees as possible, all operating within the same team and taking the risky action. It is clear from Theorem 1 that her payoff is then non-monotonic in team size, increasing in team size until some threshold, at which the team will switch from risky to safe behavior (see Figure 4).

Given this payoff function, the owner would have an incentive to manipulate the switching threshold, and hence her maximum payoff, by designing a work environment with a high $k$, making it possible for larger teams to play the risky action in long run equilibrium. As noted below Corollary 2 , a principal could adjust $k$ by providing more opportunities for workers to communicate and share intentions, perhaps through changes in the physical space or through more social events. This suggests a complementarity between different aspects of a firm's organizational and architectural design akin to the complementarity in managerial practices observed by Ichniowski et al. (1997) and Gant et al. (2002b).

Moreover, formal organizational structures will often foster informal communication. By examining messages sent in a large organization, Kleinbaum et al. (2008) found that formal structures are a significant determinant of the frequency of informal communication between workers. This is important, as frequent communication, even at an informal level, is a vital catalyst for the emergence of behavioral norms within an organization. ${ }^{7}$ A historical example of the exploitation of this complementarity between formal and informal structures was the establishment of the General Technical Committee at General Motors by Alfred P. Sloan. The committee brought together engineers from different parts of the organization. It had no formal agenda, but existed merely as 'a place to bring these men together under amicable circumstances for the exchange of information and the ironing out of differences' (Sloan, 1964, p. 106). This suggests that the real benefit of certain formal relationships in a firm may well be the informal collaboration they spawn.

There may be times when a firm owner wants a small team to avoid taking risks. In such cases, the opposite prescription to that discussed above would apply. The firm owner should structure the workplace to reduce $k$ and reduce opportunities for collaborative interaction between employees. In summary, we emphasize the important point that an owner will design a firm's organizational architecture with its employees' social interaction, and the strategic outcomes that follow, in mind.

\footnotetext{
${ }^{7}$ Johnson et al. (1994) even finds that informal communication is more effective than formal communication, with the recipients finding informal messages more salient than formal ones.
} 


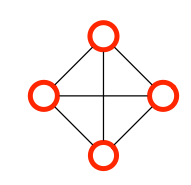

$|N|=4, X=N$

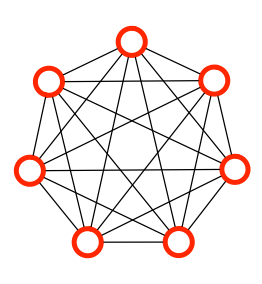

$|N|=7, X=N$

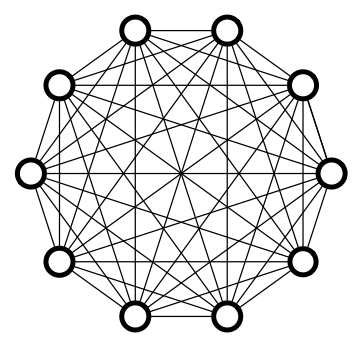

$|N|=10, X=\emptyset$

Figure 4: Teams of size $|N|=4,7,10 . q=9 / 10, k=7$. Employees circled in red play the risky action in long run equilibrium. Employees circled in black play the safe action in long run equilibrium.

\section{Vertical and horizontal structure in firms}

As a quick glance at any corporate annual report indicates, organizational structures in firms are typically more complex than a single team with everyone working together. Two key aspects of an organization's structure are the length of its hierarchy and each supervisor's span of control, which indicates how many subordinates each supervisor has. In this section we show that hierarchical structures can significantly affect the actions adopted by different departments/divisions/teams in an organization; the actions of a team depend on the balance of external and internal influence. Therefore, a principal or owner can manipulate the actions taken through separate divisions throughout a firm by altering the connective structures within the organization and the size of various teams. We are also able to comment on the recent trend in firms towards delayering - the shortening of hierarchies, the increasing span of control of CEOs (Guadalupe and Wulf, 2007; Rajan and Wulf, 2006) and the increasing size of the senior executive group (Guadalupe et al., 2014).

To consider more complex structures with multiple teams, let the set of employees $N$ be partitioned into teams $T_{m}, m=1, \ldots, \bar{m}$.

$$
N=\bigcup_{i=1}^{\bar{m}} T_{m}, \quad T_{m} \cap T_{n}=\emptyset \text { for } m \neq n .
$$

Let $m(i)$ denote the team to which employee $i$ belongs. It is assumed that an employee always interacts with members of his own team. That is, $\left(T_{m(i)} \backslash\{i\}\right) \subseteq \triangle_{i}$

Each team has its own watercooler and we again limit the number of employees who can fit around the watercooler to a maximum of $k$. Any set of no more than $k$ employees within a team has some opportunity to form shared intentions at the watercooler. 
Assumption 3. $\mathcal{C}=\left\{C \subseteq T_{m}: 1 \leq m \leq \bar{m},|C| \leq k\right\}$ for some $k \in \mathbb{N}_{+}$.

For simplicity's sake, we assume that every member of every team interacts with at most a single employee outside of the team. Similar results hold if this assumption is relaxed, but this simplification allows us to present results with a minimum of distracting caveats.

Assumption 4. For all $i,\left|\triangle_{i} \backslash T_{m(i)}\right| \leq 1$.

Then, within a given team, we can derive a similar result to Theorem 1, with the conditions on the inequalities strengthened so that $k$ becomes $k+1$ and $k-2$ becomes $k-3$ in the numerators of the relevant fractions. Note that the following theorem only concerns teams that cannot all fit around the watercooler $(|T|>k)$. That is, it does not apply to small teams, only to large and medium size teams.

Theorem 2. Under Assumptions 3 and 4, for a given team $T,|T|>k$, in any long run equilibrium $X$ :

$$
\begin{aligned}
& |T|>\frac{k+1}{2 q-1} \quad \Longrightarrow \quad T \subseteq N \backslash X, \\
& |T| \leq \frac{k-3}{2 q-1} \quad \Longrightarrow \quad T \subseteq X .
\end{aligned}
$$

So all long run equilibria involve medium-size teams playing the risky action and large teams playing the safe action. If a firm contains both types of teams, then a diversity of behavior persists within the firm. This diversity of behavior is driven by nothing more than the localized nature of interaction within the corporate structure and the ability of employees within teams to share intentions and adjust their behavior in a collaborative manner.

Now, consider small teams such that the whole team can meet at the watercooler $(|T| \leq k)$. If such a team is large enough, it ignores whatever its neighbors are doing and coordinates on the risky action in any long run equilibrium. If the team is smaller still, then its actions in long run equilibrium will depend on those of its neighbors. If no player in such a team has a neighbor outside of the team who plays the safe action, then all members of the team will play the risky action. However, if even a single member of the team has a neighbor outside of the team who plays the safe action, then members of the team are driven to do likewise. For a set of employees $C \subseteq N$, we let $\triangle_{C}$ denote the set of employees outside of $C$ who are neighbors of an employee in $C$, that is $\triangle_{C}:=\bigcup_{i \in C} \triangle_{i} \backslash C$.

Theorem 3. Under Assumptions 3 and 4, for a given team $T,|T| \leq k$, in any long run equilibrium $X$ :

$$
|T| \geq \frac{1}{1-q} \quad \Longrightarrow T \subseteq X,
$$




$$
\begin{aligned}
& |T|<\frac{1}{1-q}, \Delta_{T} \subseteq X \quad \Longrightarrow \quad T \subseteq X, \\
& |T|<\frac{1}{1-q}, \Delta_{T} \nsubseteq \subseteq X \quad \Longrightarrow \quad T \subseteq N \backslash X .
\end{aligned}
$$

So coalitional behavior can lead to heterogeneous choices by teams within a firm depending on their size. This effect is not necessarily monotonic. Large teams play the safe action, medium-size teams the risky action. In the absence of neighbors, small teams would also play the risky action, but the presence of neighbors playing safe is enough incentive for very small teams to also choose the safe action.

We can consider these effects as being driven by the relative strengths of the internal pressure from within a team and external pressure from interaction with outsiders. Both medium-sized and large teams are large enough to be immune to external pressure. Their culture is determined internally: the collaborative impact of the watercooler is enough to cause members of medium-sized teams to engage in risky and innovative behavior, but it is not enough to overcome the coordination problem in large teams. For the smallest teams, external pressure outweighs internal pressure and even though the coordination problem can easily be solved within the team, the presence of neighbors who play the safe action is sufficient to encourage these teams to take the safe action.

By exploiting these internal and external pressures, a firm owner or manager can manipulate the structure of the firm to achieve desired outcomes. If the manager would like a safe action to be taken by a small workgroup, she will ensure it has strong links to a division that will definitely be playing the safe action - typically a large department. On the other hand, if the manager would like a team to take risks - this group could be the firm's research group - this team should be small and either have limited links to the rest of the firm, or only links to other risk-takers in the firm. This accords with the lessons from the disruptive innovation literature. Bower and Christensen (1995) suggest that a prudent investment strategy of a large firm will avoid risky new innovations with limited current demand. Rather, it is small and hungry organizations that will take on these risky opportunities. Further, if a large firm is to be able to exploit disruptive innovation opportunities, the cited work advocates creating a small team that is isolated (and remains isolated) from the mainstream organization.

Entrepreneurs do indeed realize the potential cost of too much communication. As Slone (2013) records, the founder of Amazon.com, Jeff Bezos, has suggested

"We should be trying to figure out a way for teams to communicate less with each other, not more". 
An example of this maxim being put into practice is the Palo Alto Research Center (PARC), established by Xerox to create the innovations of the future. The PARC was deliberately geographically isolated from Xerox's headquarters and existing research laboratory in New York. Given its intended role, it was important that the PARC was separated from the main bureaucratic processes and culture of Xerox, which was conservative and focused on its traditional copier business (Regani, 2005). ${ }^{8}$

\subsection{Example: Delayering}

There has been a trend in recent decades for organizations to shorten the lengths of their hierarchies. Moreover, many of these firms have also increased the span of control of the senior management group; there has been a notable increase in the number of individuals who directly report to the CEO. While there can be other drivers for such changes - Guadalupe and Wulf (2010) emphasize the impact of product-market competition from internalization - here we use Theorems 2 and 3 to look at a possible relationship between watercooler chat and delayering.

For this purpose, let $q=3 / 4$ and $k=8$. Consider the fragment of a corporate hierarchy in Figure 5(a) that includes six teams labeled A to F. Teams A, B, C and D are all larger than $k$, meaning that it is not possible for all of the members of these teams to fit around the watercooler at once. Theorem 2 then implies that in any long run equilibrium, Team A will take the safe action and Teams B, C, D will take the risky action. Teams $\mathrm{E}$ and $\mathrm{F}$ are smaller than $k$, so that the whole team can fit around the watercooler. Each of these two teams contains a member who is connected to some member of Team C. Team E is of size 5 , larger than $1 /(1-q)=4$, so the first statement of Theorem 3 implies that in any long run equilibrium, Team E will take the risky action. Team F, on the other hand, is smaller than $1 /(1-q)$, but as it does not have any neighboring teams who take the safe action, by the second statement of Theorem 3, it will play the risky action.

\footnotetext{
${ }^{8}$ In 1970 Xerox Inc. established the PARC with the objective that it develop 'future technologies'. In an extremely innovative environment, PARC was responsible for many fundamental computing innovations such as developing the prototype $\mathrm{PC}$, the ethernet, the what-you-see-is-what-you-get computer screen, the graphical user interface, the commercial application of the mouse, page description languages and the laser printer (Kovar, 1999; Regani, 2005). The isolation from the rest of the company was intentional and lay at the heart of the laboratory's success: Charles Geschke, a researcher at PARC from 1972 until 1982 suggested that 'When George Pake ... started the lab, he realized that if he simply put a building next to the research lab in Webster [N.Y.], it would very likely [be] sort of sucked into the kind of research that Xerox had been doing historically' (Kovar, 1999). More recently, in 2002 PARC was formally separated from Xerox and incorporated as a stand-alone research entity, wholly owned by Xerox Inc. (Regani, 2005).
} 


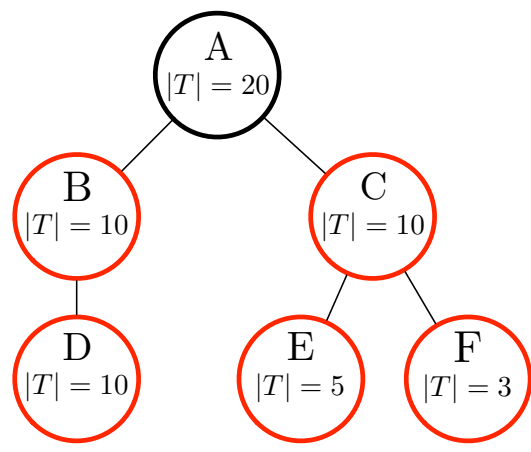

(a) Pre-delayering

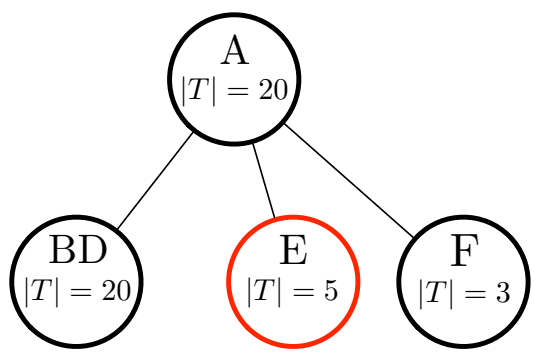

(b) Post-delayering

Figure 5: A line between two teams indicates a link between a member of each team. Teams circled in red play the risky action in long run equilibrium. The remaining teams play the safe action in long run equilibrium.

Now, consider the delayering of the firm, leading to the hierarchy in Figure 5(b). Two things have changed. Teams B and D have been merged into a single team - Team BD - and Team C (middle management) has been eliminated, so that Teams $\mathrm{E}$ and $\mathrm{F}$ are now directly connected to Team A. These changes affect the culture of the employees in the surviving teams. The merging of $\mathrm{B}$ and $\mathrm{D}$, two teams that previously played the risky action has led to Team $\mathrm{BD}$, which by Theorem 2, will play the safe action in any long run equilibrium. Delayering, in the case of Team BD, has created a unit of sufficient scale such that it will play the safe action in long run. Moreover, its size makes it immune to external pressures from Head Office in that Team BD would play safe regardless of the action taken by Team A.

The elimination of Team $\mathrm{C}$ does not affect Team $\mathrm{E}$, which is large enough that its decision to play the risky action cannot be outweighed by external influence (first statement of Theorem 3). However, Team F is now in direct contact with Head Office, which plays the safe action. It follows from the third statement of Theorem 3 that all employees in Team F will also now play the safe action. The external contact here is crucial as it allows the senior manager to switch the behavior of a small unit.

The analysis of this section shows how delayering can create opportunities for a principal to exercise her influence by creating different sized teams in her organization and linking them to create the right balance between external and internal pressures. In this way, different behavior can be generated in separate parts of an organization, whenever this is a required component of the organization's strategy. 

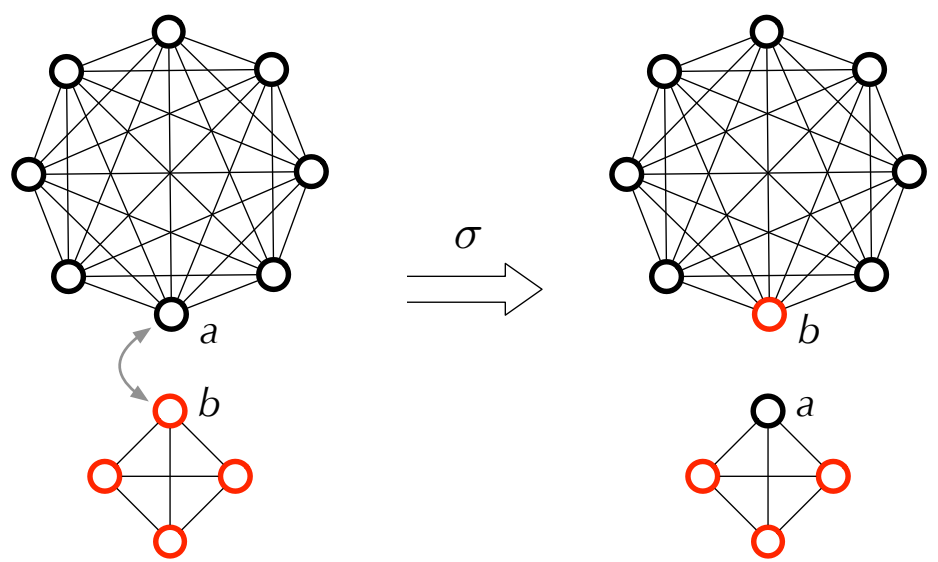

Figure 6: Two employees exchange places. Employees circled in red are playing the risky action. The remaining employees are playing the safe action.

\subsection{Example: Job rotation}

Firms might choose to rotate workers through tasks for a variety of reasons. Arya and Mittendorf (2004) note that rotation can aid in information collection from agents. Choi and Thum (2003) argue that it can help overcome boredom and reduce corruption. Here we show that rotation can act as a mechanism to allow the culture of one part of an organization to contage another part of the organization. Specifically, we show how even relatively short spans of time spent working in a small team can shape an employee's behavior. When rotated back to a larger team, the employee will, for a while, retain the behavior to which he became accustomed in the small team. The periodic arrival of such employees is enough to change the long run culture of the large team from safe to risky/innovative.

If $q=9 / 10, k=7$ and there are two independent teams of size 4 and 10 , then, as we saw in Figure 4, these teams play the risky and safe actions respectively in long run equilibrium. Here, we amend the model to that with probability given by the Poisson rate $\sigma$, two employees, one from each team, exchange places. That is, they switch teams whilst leaving their action unchanged. Figure 6 gives an example of such a switch.

Now, from any state, the state $X=N$ can be reached without mistakes in action choice. To see this, consider that the following sequence of events will occur with positive probability. First, all current members of the small team meet at the small team's watercooler, where they will agree to play the risky action. Second, the members of the small team switch places, one by one, with members of the large team. This gives at least four members of the large team who are now playing the risky action. Third, the other six members of the large team meet at the large team's watercooler and agree 
to switch to the risky action. They are happy to do this as the remaining four members of the team are already playing the risky action. Finally, the new members of the small team all meet at the small team's watercooler and switch to the risky action. We have reached the state $X=N$. All employees are playing the risky action.

Furthermore, from $X=N$, any group of employees who meet at a watercooler will agree to continue playing the risky action. That is, mistakes in strategy choice are required to leave this state. Therefore, when the mistake rate $\varepsilon$ is low, all employees will take the risky action almost all of the time. That is, $X=N$ is the unique long run equilibrium.

\section{Concluding comments}

While the boundaries of a firm are defined by its physical assets (Hart and Moore, 1990), social interactions between workers characterize the way things get done in an organization. Workers idly sharing scuttlebutt around the watercooler might seem like the bane of an employer's life, but these informal interactions could engender collective actions that enhance firm productivity. This paper has examined how a manager can tinker with an organization's structure and the physical work environment to harness workers' informal interactions for the firm's advantage.

Although the direct application considered in this paper is the design of a firm, it is clear that adaptive/evolutionary models that incorporate some degree of collective agency should also be applicable to other problems in applied economics. In particular, the implications of collective agency may be of particular importance whenever formal structures in an organization can facilitate informal interactions. This is true for academic conferences, where informal interactions are typically of more import than organized presentations, and also for diplomacy, where formal meetings are accompanied by informal, less structured, discussions in which parties are often more able to find common ground and create shared intentions.

\section{References}

Ambrus, A., 2009. Theories of coalitional rationality. Journal of Economic Theory 144, $676-695$.

Angus, S.D., Newton, J., 2015. Emergence of shared intentionality is coupled to the advance of cumulative culture. PLoS Comput Biol 11, e1004587.

Arrow, K.J., 1994. Methodological individualism and social knowledge. American Economic Review 84, 1-9.

Arya, A., Mittendorf, B., 2004. Using Return Polices to Elicit Retailer Information. RAND Journal of Economics 35, 617-630.

Aumann, R., 1959. Acceptable points in general cooperative n-person games, in contributions to the theory of games iv, (A. W. Tucker and R. D. Luce, eds.). Princeton University Press , 287-324. 
Bacharach, M., 2006. Beyond individual choice: teams and frames in game theory. Princeton University Press.

Baker, G., Gibbons, R., Murphy, K., 1999. Informal authority in organizations. Journal of Law, Economics and Organization 15, 56-73.

Bandiera, O., Barankay, I., Rasul, I., 2005. Social preferences and the response to incentives: Evidence from personnel data. Quarterly Journal of Economics 122, 729 773.

Baron-Cohen, S., 1994. From attention-goal psychology to belief-desire psychology, in: Baron-Cohen, S., Tager-Flusberg, H., Cohen, D.J. (Eds.), Understanding other minds: Perspectives from autism.. Oxford University Press.

Bernheim, B.D., Peleg, B., Whinston, M.D., 1987. Coalition-proof nash equilibria i. concepts. Journal of Economic Theory 42, 1-12.

Bower, J.L., Christensen, C.M., 1995. Disruptive technologies: Catching the wave. Harvard Business Review January-February.

Call, J., Tomasello, M., 1999. A nonverbal false belief task: The performance of children and great apes. Child development 70, 381-395.

Carpenter, M., Akhtar, N., Tomasello, M., 1998a. Fourteen-through 18-month-old infants differentially imitate intentional and accidental actions. Infant Behavior and Development 21, 315-330.

Carpenter, M., Nagell, K., Tomasello, M., Butterworth, G., Moore, C., 1998b. Social cognition, joint attention, and communicative competence from 9 to 15 months of age. Monographs of the society for research in child development, i-174.

Carvalho, J.P., 2013. Veiling. The Quarterly Journal of Economics 128, 337-370.

Chaganti, R.S., Mahajan, V., Sharma, S., 1985. Corporate board size, composition and corporate failures in retailing industry[1]. Journal of Management Studies 22, 400-417.

Cheng, S., 2008. Board size and the variability of corporate performance. Journal of Financial Economics 87, $157-176$.

Choi, J.P., Thum, M., 2003. The dynamics of corruption with the ratchet effect. Journal of Public Economics 87, 427-443.

Chong, J.K., Camerer, C.F., Ho, T.H., 2006. A learning-based model of repeated games with incomplete information. Games and Economic Behavior 55, 340-371.

Coles, J.L., Daniel, N.D., Naveen, L., 2008. Boards: Does one size fit all? Journal of Financial Economics 87, 329 - 356.

Cook, J., 2012. How google motivates their employees with rewards and perks. http://thinkingleaders.com .

Cooper, R., DeJong, D.V., Forsythe, R., Ross, T.W., 1992. Communication in Coordination Games. The Quarterly Journal of Economics 107, 739-771.

Cournot, A.A., 1838. Recherches sur les principes mathématiques de la théorie des richesses par Augustin Cournot. chez L. Hachette.

Eisenberg, T., Sundgren, S., Wells, M.T., 1998. Larger board size and decreasing firm value in small firms. Journal of Financial Economics 48, $35-54$.

Evans, S., 2015. No phones on desks as kpmg spends $\$ 7 \mathrm{~m}$ on agile workspace for digital age. Australian Financial Review 6 August.

Foster, D., Young, H.P., 1990. Stochastic evolutionary game dynamics. Theoretical Population Biology 38, 219-232.

Freidlin, M.I., Wentzell, A.D., 1984. Random perturbations of dynamical systems, ISBN 9780387983622430 pp., 2nd ed.(1998). Springer .

Gant, J., Ichniowski, C., Shaw, K., 2002a. Social capital and organizational change in high-involvement and traditional work organizations. Journal of Economics and Management Strategy 11, 289-328.

Gant, J., Ichniowski, C., Shaw, K., 2002b. Social capital and organizational change 
in high-involvement and traditional work organizations. Journal of Economics and Management Strategy 11, 289-328.

Gibbons, R., Henderson, R., 2013. What do managers do?, in: Gibbons, R., Roberts, J. (Eds.), Handbook of Organizational Economics. Princeton University Press, pp. 680-731.

Guadalupe, M., Li, H., Wulf, J., 2014. Who lives in the c-suite? organizational structure and the division of labor in top management. Management Science 60, 824-844.

Guadalupe, M., Wulf, J., 2007. The flattening firms and product market competition: The effects of trade costs and liberalization. American Economic Journal: Applied .

Guadalupe, M., Wulf, J., 2010. The flattening firm and product market competition: The effect of trade liberalization on corporate hierarchies. American Economic Journal: Applied Economics 2, 105-27.

Hart, O.D., Moore, J., 1990. Property rights and the nature of the firm. Journal of Political Economy 98.

Ichniowski, C., Shaw, K., Prennushi, G., 1997. The effects of human resource management practices on productivity: A study of steel finishing lines. American Economic Review 86, 291-313.

Jackson, M.O., Watts, A., 2002. The evolution of social and economic networks. Journal of Economic Theory 106, 265-295.

Johnson, D.J., Donohue, W.A., Atkin, C.K., 1994. Differences between formal and informal communication channels. The Journal of Business Communication 31, 111122.

Kandel, E., Lazear, E.P., 1992. Peer pressure and partnerships. Journal of Political Economy 100, 801-817.

Kandori, M., Mailath, G.J., Rob, R., 1993. Learning, mutation, and long run equilibria in games. Econometrica 61, 29-56.

Klaus, B., Klijn, F., Walzl, M., 2010. Stochastic stability for roommate markets. Journal of Economic Theory 145, $2218-2240$.

Klaus, B., Newton, J., 2016. Stochastic stability in assignment problems. Journal of Mathematical Economics 62, $62-74$.

Kleinbaum, A.M., Stuart, T.E., Tushman, M.L., 2008. Communication (and coordination?) in a modern, complex organization. HBS Working Paper .

Konishi, H., Ray, D., 2003. Coalition formation as a dynamic process. Journal of Economic Theory 110, 1-41.

Kovar, J.F., 1999. 1999 industry hall of fame: Xerox parc. Computer Reseller News 868, 48-49.

Kraut, R.E., Fish, R.S., Root, R.W., Chalfonte, B.L., Oskamp, I.S., 1990. Informal communication in organizations: Form, function, and technology, in: Oskamp, I.S., Spacapan, S. (Eds.), Human Reactions to Technology: The Claremont Symposium on Applies Social Psychology.

Levin, J., 2003. Relational incentive contracts. The American Economic Review 93, $835-857$.

Li, J., Matouschek, N., Powell, M., 2014. The burden of past promises. Northwestern University.

Nax, H.H., Pradelski, B.S.R., 2014. Evolutionary dynamics and equitable core selection in assignment games. International Journal of Game Theory 44, 903-932.

Newton, J., 2012a. Coalitional stochastic stability. Games and Economic Behavior 75, $842-54$.

Newton, J., 2012b. Recontracting and stochastic stability in cooperative games. Journal of Economic Theory 147, 364-81.

Newton, J., 2015. Shared intentions: the evolution of collaboration. Working Papers 2015-05. University of Sydney, School of Economics. 
Newton, J., Angus, S.D., 2015. Coalitions, tipping points and the speed of evolution. Journal of Economic Theory 157, 172 - 187.

Newton, J., Sawa, R., 2015. A one-shot deviation principle for stability in matching problems. Journal of Economic Theory 157, $1-27$.

Peleg, B., Sudholter, P., 2003. Introduction to the theory of cooperative games, ISBN 1-4020-7410-7 378 pages. Kluwer Academic, Boston .

Peski, M., 2010. Generalized risk-dominance and asymmetric dynamics. Journal of Economic Theory 145, $216-248$.

Rajan, R.G., Wulf, J., 2006. The flattening firm: Evidence from panel data on the changing nature of corporate hierarchies. The Review of Economics and Statistics 88, 759-773.

Regani, S., 2005. Xerox PARC: Innovation without profit? ICMR Center for Management Research 2005.

Sawa, R., 2014. Coalitional stochastic stability in games, networks and markets. Games and Economic Behavior 88, 90-111.

Serrano, R., Volij, O., 2008. Mistakes in cooperation: the stochastic stability of edgeworth's recontracting. Economic Journal 118, 1719-1741.

Sloan, A.P., 1964. My Years with General Motors. Doubleday.

Slone, B., 2013. The Everything Store: Jeff Bezos and the Age of Amazon. Little, Brown and Company.

Srikant, P., 2014. Why google is the best place to work for? Amity Research Case Study 2014.

Stross, R.E., 1996. The Microsoft Way: The Real Story Of How The Company Outsmarts Its Competition. Basic Books.

Tomasello, M., 2014. A natural history of human thinking. Harvard University Press.

Tomasello, M., Carpenter, M., Call, J., Behne, T., Moll, H., 2005. Understanding and sharing intentions: The origins of cultural cognition. Behavioral and brain sciences 28, 675-691.

Tomasello, M., Rakoczy, H., 2003. What makes human cognition unique? from individual to shared to collective intentionality. Mind \& Language 18, 121-147.

Van Huyck, J.B., Battalio, R.C., Beil, R.O., 1990. Tacit coordination games, strategic uncertainty, and coordination failure. The American Economic Review , 234-248.

Weber, R., Camerer, C., Rottenstreich, Y., Knez, M., 2001. The illusion of leadership: Misattribution of cause in coordination games. Organization Science 12, 582-598.

Wellman, H.M., Bartsch, K., 1994. Before belief: Childrens early psychological theory. Childrens early understanding of mind: Origins and development, 331-354.

Wellman, H.M., Cross, D., Watson, J., 2001. Meta-analysis of theory-of-mind development: the truth about false belief. Child development 72, 655-684.

Yermack, D., 1996. Higher market valuation of companies with a small board of directors. Journal of Financial Economics 40, 185 - 211.

Young, H.P., 1993. The evolution of conventions. Econometrica 61, 57-84.

Young, H.P., 1998. Individual strategy and social structure. Princeton University Press.

Young, H.P., Burke, M.A., 2001. Competition and custom in economic contracts: A case study of illinois agriculture. American Economic Review 91, 559-573. 


\section{Appendix A. Proofs}

We consider a discrete time Markov chain associated with the process of the model. We consider the chain derived from observing the process at $t \in \mathbb{N}_{0}$. Note that for this chain, any transition probability is of the order of $\varepsilon^{r}$ for some $r \in \mathbb{N}_{0}$, with $r$ being equal to the lowest number of perturbations required to effect the transition under the original process. We denote the $t$-period Markov transition probability from state $S$ to state $T$ by $P_{k, q, \varepsilon}^{t}(S, T)$.

We apply the results of Freidlin and Wentzell (1984) as adapted by Foster and Young (1990); Kandori et al. (1993); Young (1993). For $S, T \subseteq$ $N$, define the resistance $r(S, T)$ so that the most probable transition from state $S$ to state $T$ occurs with probability of order $\varepsilon^{r(S, T)}$.

$$
r(S, T)=\min \left\{r \in \mathbb{R}_{+}: \exists t \in \mathbb{N}_{+}: \lim _{\varepsilon \rightarrow 0} \frac{P_{k, q, \varepsilon}^{t}(S, T)}{\varepsilon^{r}}>0 .\right\}
$$

Note that strategic complementarity and the absence of ties imply that any communicating class of the process with $\varepsilon=0$ is a singleton. This implies that any communicating class $\{S\}$ is a rest point of the dynamic with $\varepsilon=0$, such that $P_{k, q, 0}(S, S)=1$.

Proof of Theorem 1. The process with $\varepsilon=0$ has at most two communicating classes. $\{N\}$ is always a communicating class, that is $P_{k, q, 0}(N, N)=1$. $\{\emptyset\}$ is a communicating class if and only if If $(k-1) /(|N|-1)<q$. By Young (1993), any long run equilibrium must be part of a communicating class of the chain with $\varepsilon=0$. Furthermore, if $r(N, \emptyset)>r(\emptyset, N)$, then $N$ is the unique long run equilibrium. If $r(\emptyset, N)>r(N, \emptyset)$, then $\emptyset$ is the unique long run equilibrium.

Now, $r(N, \emptyset)$ is the lowest integer satisfying

$$
q>\frac{|N|-1-r(N, \emptyset)}{|N|-1}
$$

which rearranges to

$$
r(N, \emptyset)>(1-q)(|N|-1) \quad \text { so } \quad r(N, \emptyset)=\lfloor(1-q)(|N|-1)+1\rfloor .
$$

$r(\emptyset, N)$ is the lowest integer satisfying

$$
q \leq \frac{(k-1)+r(\emptyset, N)}{|N|-1}
$$

which rearranges to

$$
r(\emptyset, N) \geq q(|N|-1)-k+1 \quad \text { so } \quad r(\emptyset, N)=\lceil q(|N|-1)-k+1\rceil .
$$


Simple manipulation then shows that:

$$
\begin{array}{lll}
|N|-1>\frac{k}{2 q-1} & \Longrightarrow & r(\emptyset, N)>r(N, \emptyset) \\
|N|-1 \leq \frac{k-2}{2 q-1} & \Longrightarrow & r(N, \emptyset)>r(\emptyset, N)
\end{array}
$$

Proof of Theorem 2. For any communicating class $\{X\}$, for any two employees in the same team, $i, j \in T$, it cannot be that $i \in X$ and $j \notin X$, as this would imply $q_{j}(X) \geq q_{i}(X)$ and as $i \in X$ implies $q_{i}(X) \geq q$, we have that $q_{j}(X) \geq q$, so that when $j$ gets the opportunity to update his strategy he will switch to the risky action, contradicting $\{X\}$ being a communicating class. So in any communicating class, and hence in any long run equilibrium, members of the same team play the same action.

Similar manipulation to that in the proof of Theorem 1 gives

$$
\begin{aligned}
& |T|>\frac{k+1}{2 q-1} \Longrightarrow \min _{Y: T \subseteq N \backslash Y} \min _{Z: T \subseteq Z} r(Y, Z)>\max _{Y: T \subseteq Y} \min _{Z: T \subseteq N \backslash Z} r(Y, Z) \\
& |T| \leq \frac{k-3}{2 q-1} \Longrightarrow \min _{Y: T \subseteq Y} \min _{Z: T \subseteq N \backslash Z} r(Y, Z)>\max _{Y: T \subseteq N \backslash Y} \min _{Z: T \subseteq Z} r(Y, Z)
\end{aligned}
$$

In words, $|T|>(k+1) /(2 q-1)$ implies that the least resistance in moving from any state at which $T$ plays the safe action to some state at which $T$ plays the risky action is greater than the maximum resistance of any move in the opposite direction. By Freidlin and Wentzell (1984); Young (1993), this implies that at any long run equilibrium $X$, we have $T \subseteq N \backslash X$. Similarly, $|T| \leq(k-3) /(2 q-1)$ implies that at any long run equilibrium $X$, we have $T \subseteq X$.

Proof of Theorem 3. Consider the chain with $\varepsilon=0$, starting from a conjectured long run equilibrium state $X$.

Assume $T \nsubseteq X$. If $|T| \geq 1 /(1-q)$, then $(|T|-1) /|T| \geq q$, so when $T$ gets an opportunity to update as a coalition, all members would switch from the safe action to the risky action. So $X$ is not part of any communicating class of the chain with $\varepsilon=0$ and is therefore not a long run equilibrium.

Again, assume $T \nsubseteq X$. If $|T|<1 /(1-q)$ and $\Delta_{T} \subseteq X$, then when $T$ gets an opportunity to update as a coalition, all members would switch from the safe action to the risky action. So $X$ cannot be a long run equilibrium.

Assume $T \subseteq X$. If $|T|<1 /(1-q)$ and $\Delta_{T} \nsubseteq X$, then when $T$ gets an opportunity to update as a coalition, as $(|T|-1) /|T|<q$, there exists $i \in T$ who will switch to the safe action. Consequently, it follows that $X$ cannot be a long run equilibrium. 